\title{
Approximate solution for frequency synchronization in a finite-size Kuramoto model
}

\author{
Chengwei Wang, ${ }^{1}$ Nicolás Rubido, ${ }^{1,2}$ Celso Grebogi, ${ }^{1}$ and Murilo S. Baptista ${ }^{1}$ \\ ${ }^{1}$ Institute for Complex Systems and Mathematical Biology, University of Aberdeen, King's College, AB24 3UE Aberdeen, United Kingdom \\ ${ }^{2}$ Instituto de Física, Facultad de Ciencias, Universidad de la República, Iguá 4225, 11400 Montevideo, Uruguay
}

(Received 16 June 2015; published 8 December 2015)

\begin{abstract}
Scientists have been considering the Kuramoto model to understand the mechanism behind the appearance of collective behavior, such as frequency synchronization (FS) as a paradigm, in real-world networks with a finite number of oscillators. A major current challenge is to obtain an analytical solution for the phase angles. Here, we provide an approximate analytical solution for this problem by deriving a master solution for the finite-size Kuramoto model, with arbitrary finite-variance distribution of the natural frequencies of the oscillators. The master solution embodies all particular solutions of the finite-size Kuramoto model for any frequency distribution and coupling strength larger than the critical one. Furthermore, we present a criterion to determine the stability of the FS solution. This allows one to analytically infer the relationship between the physical parameters and the stable behavior of networks.
\end{abstract}

DOI: 10.1103/PhysRevE.92.062808

PACS number(s): 89.75.Fb, 05.45.Xt

\section{INTRODUCTION}

Networks of coupled oscillators provide a pragmatic model to describe basic behavior of natural and technological systems, such as biological networks [1,2], social networks [3], computer networks [4,5], and power grids [6,7]. A significant phenomenon emerging from coupled oscillators is the synchronization of the oscillators' rhythms [8-11]. Since 1958, when Norbert Wiener [12] suggested the presence of collective behavior of neurons in the brain, finding the mechanism and the conditions for the appearance of synchronization in complex networks has attracted the attention of many scientists. Kuramoto [13-15] proposed a mathematically tractable model by considering a network of phase oscillators coupled with an all-to-all topology through a sine function, with each oscillator possessing a constant natural frequency. Recently, scientists devoted great effort to understand the collective behavior and synchronization of the Kuramoto model and its connections to natural systems [16-21].

However, most of the existing analytical results have considered networks with an infinite number of oscillators and have imposed restrictions on the distributions of natural frequencies of oscillators. To the best of our knowledge, no analytical work has been proposed that allows one to determine the synchronous solution of the phase angles for the finite-size Kuramoto model without restriction on natural frequencies with finite-variance distribution. Although the conditions for the set of natural frequencies that provides stable synchronization is obtained in [22], the solution for the phase angles is not given. But, solving the phase angles is of fundamental significance in real-world systems. For example, in power grids [6,7], which can be described by a Kuramotolike model [23], one is not only interested in understanding under which conditions the frequency synchronization (FS) among oscillators emerges, but also in knowing the phase angles after synchronization. The phase angles are important variables for monitoring generators and developing control strategies for the power grid. Similarly, in the research of Josephson junctions [24,25], a Kuramoto-type model has been widely considered [26-30] to explain the synchronization phenomenon in the junctions, and the phase angles of a syn- chronous state contain the information of the wave-function phase difference across every Josephson junction.

In this work, we present an analytical method to approximately calculate the phase angles for the finite-size Kuramoto model when the frequencies are synchronized. Compared to other works, our method does not require any restriction on the distribution of the natural frequencies of oscillators. Our solution, shown in Eq. (24), directly links the FS solution and the physical parameters of the network. Remarkably, the solution is independent of the network size, and only depends on the natural frequencies and the coupling strength. In addition, we provide an approximate criterion, shown in Eq. (26), to analytically predict whether a finite number of oscillators are able to emerge into a stable FS, even without knowing the FS solution explicitly.

\section{THE FINITE-SIZE KURAMOTO MODEL}

We use $\overrightarrow{1}_{N}\left(\overrightarrow{0}_{N}\right)$ to denote the $N \times 1$ vector with all elements equal to $1(0), \mathcal{I}_{N}$ to indicate the index set $\{1,2, \ldots, N\}$, and $\mathbb{R}$ to represent the set of real numbers. Given a vector $\vec{\alpha}$ with $N$ elements, we use $\langle\alpha\rangle=\frac{1}{N} \sum_{i=1}^{N} \alpha_{i}$ to denote the mean value of $\vec{\alpha}$.

The finite-size Kuramoto model, describing the dynamical behavior of $N$ phase oscillators in an all-to-all network, is given by the equation

$$
\dot{\Theta}_{i}=\Omega_{i}+\frac{K}{N} \sum_{j=1}^{N} \sin \left(\Theta_{j}-\Theta_{i}\right), \quad \forall i \in \mathcal{I}_{N},
$$

where $N$ is a finite positive integer number, $K$ is the coupling strength, and the $N \times 1$ vectors, $\vec{\Omega}=\left[\Omega_{1}, \Omega_{2}, \ldots, \Omega_{N}\right]^{T}$ and $\Theta=\left[\Theta_{1}, \Theta_{2}, \ldots, \Theta_{N}\right]^{T}$, denote the natural frequencies and instantaneous phases of the oscillators, respectively. We rewrite Eq. (1) in a rotating frame by letting $\vec{\theta} \equiv \vec{\Theta}-\overrightarrow{1}_{N}\langle\Omega\rangle t$ and $\vec{\omega} \equiv \vec{\Omega}-\overrightarrow{1}_{N}\langle\Omega\rangle$, such that $\langle\omega\rangle=\frac{1}{N} \sum_{i=1}^{N}\left(\Omega_{i}-\langle\Omega\rangle\right)=$ 0 . Therefore, if $g(\Omega)$ represents a function of an arbitrary distribution with finite variance for the natural frequencies in Eq. (1), with a mean value $\langle\Omega\rangle$ which is not necessarily equal to zero, then the distribution $g(\omega)$ in the rotating frame always 
has a mean value $\langle\omega\rangle=0$. We can change Eq. (1) into the rotating frame without losing its properties, namely,

$$
\dot{\theta}_{i}=\omega_{i}+\frac{K}{N} \sum_{j=1}^{N} \sin \left(\theta_{j}-\theta_{i}\right), \quad \forall i \in \mathcal{I}_{N} .
$$

The order parameter $r \in[0,1]$ is defined by $[13,14]$

$$
r e^{i \psi}=\frac{1}{N} \sum_{j=1}^{N} e^{i \theta_{j}}, \quad \forall j \in \mathcal{I}_{N},
$$

where $r$ and $\psi$ are calculated by equating the real and imaginary parts in Eq. (3), namely,

$$
r=\sqrt{\left(\frac{1}{N} \sum_{j=1}^{N} \sin \theta_{j}\right)^{2}+\left(\frac{1}{N} \sum_{j=1}^{N} \cos \theta_{j}\right)^{2}}
$$

and

$$
\psi=\arctan \left(\frac{\sum_{j=1}^{N} \sin \theta_{j}}{\sum_{j=1}^{N} \cos \theta_{j}}\right),
$$

where we exclude the case that $\sum_{j=1}^{N} \cos \theta_{j}=0$. We note that $\psi$ is not the average phase in a network with finite oscillators (i.e., $\psi \neq\langle\theta\rangle$ ). This is different from the situation with infinite number of oscillators [31], where $\psi=\langle\theta\rangle$ is the global mean field.

Multiplying $e^{-i \theta_{i}}$ to both sides of Eq. (3) and equating the imaginary part, we have

$$
r \sin \left(\psi-\theta_{i}\right)=\frac{1}{N} \sum_{j=1}^{N} \sin \left(\theta_{j}-\theta_{i}\right),
$$

which results in

$$
\dot{\theta}_{i}=\omega_{i}+K r \sin \left(\psi-\theta_{i}\right), \quad \forall i \in \mathcal{I}_{N} .
$$

The oscillators described by Eq. (2) emerge into FS if [32]

$$
\dot{\theta}_{i}-\dot{\theta}_{j}=0 \quad \text { as } t \rightarrow \infty, \quad \forall i, j \in \mathcal{I}_{N} .
$$

We have, from Eq. (2), that $\sum_{i=1}^{N} \dot{\theta}_{i}=\sum_{i=1}^{N} \omega_{i}=N\langle\omega\rangle$. Since $\langle\omega\rangle=0$, to satisfy Eq. (8) we require that $\dot{\vec{\theta}}=\overrightarrow{1}_{N}\langle\omega\rangle=$ $\overrightarrow{0}_{N}$. Thus, in the rotating framework, to solve the finite-size Kuramoto model in FS is to solve

$$
\dot{\theta}_{i}=\omega_{i}+\frac{K}{N} \sum_{j=1}^{N} \sin \left(\theta_{j}-\theta_{i}\right),
$$

subject to: $\dot{\theta}_{i}=0, \quad \forall i \in \mathcal{I}_{N}$.

In general, the finite-size Kuramoto model reaches FS if the coupling strength is larger than a critical value, $K_{C}$ [21]. Verwoerd and Mason [8] provided an algorithm to exactly calculate $K_{C}$ from

$$
K_{C}=\frac{u}{\frac{1}{N} \sum_{j=1}^{N} \sqrt{1-\left(\frac{\omega_{j}}{u}\right)^{2}}},
$$

where $u$ is calculated from $2 \sum_{j=1}^{N} \sqrt{1-\left(\omega_{j} / u\right)^{2}}=\sum_{j=1}^{N} 1 /$ $\sqrt{1-\left(\omega_{j} / u\right)^{2}}$.

\section{SOLUTION OF THE FINITE-SIZE KURAMOTO MODEL}

The stability of the FS solution of the finite-size Kuramoto model can be studied by the Lyapunov function,

$$
E=\frac{1}{2} \dot{\vec{\theta}}^{T} \dot{\vec{\theta}},
$$

where the time derivative of $E$ along the trajectories of Eq. (2) is $\dot{E}=-\frac{K}{N} \sum_{1 \leqslant i<j \leqslant N}^{N} \cos \left(\theta_{i}-\theta_{j}\right)\left(\dot{\theta}_{i}-\dot{\theta}_{j}\right)^{2}$. A sufficient condition for the stability of the FS solution is that $\dot{E}<0$, implying $\cos \left(\theta_{i}-\theta_{j}\right)>0, \forall i, j \in \mathcal{I}_{N}$. This means that $\left|\theta_{i}-\theta_{j}\right|<\frac{\pi}{2}, \forall i, j \in \mathcal{I}_{N}$. We denote the stable critical coupling strength of the finite-size Kuramoto model by $K_{S}$ defined as

$K_{S}:=\min \left\{K: K \geqslant K_{C},\left|\theta_{i}-\theta_{j}\right|<\frac{\pi}{2}, \forall i, j \in \mathcal{I}_{N}\right\}$.

Thus, as $t \rightarrow \infty$, the oscillators are attracted to the stable FS if $K \geqslant K_{S}$. We define $K_{S} \geqslant K_{C}$, since $K=K_{C}$ only ensures the existence of the FS solution [8], but $K \geqslant K_{S}$ provides a condition for its stability based on the Lyapunov function. The existence of $K_{S}$ has been studied in [9,32], and [21].

Given $K=K^{*} \geqslant K_{S}$, assume $\vec{\theta}^{s}$ is a stable solution of the finite-size Kuramoto model in FS. Then, $\vec{\theta}^{\xi}=\vec{\theta}^{s}+\overrightarrow{1}_{N} \xi, \forall \xi \in$ $\mathbb{R}$ is also a stable solution corresponding to $K=K^{*}$, since the phase differences are independent of $\xi$. This means that there are an infinite number of stable solutions for the finite-size Kuramoto model in a certain FS state. We use $\mathbb{S}_{K^{*}}^{\infty}$, corresponding to $K=K^{*}$, to denote the infinite-dimensional stable solution space. Actually, $\vec{\theta}=\overrightarrow{1}_{N} \xi, \forall \xi \in \mathbb{R}$, is the homogeneous solution of Eq. (9), obtained by setting the nonhomogeneous terms to be zero, i.e., $\vec{\omega}=\overrightarrow{0}_{N} \cdot \vec{\theta}^{s}$ and $\vec{\theta} \xi$ are particular solutions of the non-homogeneous Eq. (9). Our goal is to find one of the particular solutions, which can be analytically expressed, such that Eq. (9) is analytically solvable.

We define the master solution of Eq. (9) as

$$
\vec{\phi}^{*}=\vec{\theta}^{\xi}-\overrightarrow{1}_{N} \psi^{\xi} \in \mathbb{S}_{K^{*}}^{\infty},
$$

where $\psi^{\xi}$ is calculated from Eq. (5) as $\vec{\theta}=\vec{\theta}$. There are three characteristics for which we call $\vec{\phi}^{*}$ the master solution: (i) $\vec{\phi}^{*}$ is analytically expressible; (ii) $\vec{\phi}^{*}$ is identical $\forall \xi \in \mathbb{R}$, i.e., $\vec{\phi}^{*}$ is independent of $\xi$; and (iii) $\psi^{*} \equiv 0$, where $\psi^{*}$ is calculated from Eq. (5) as $\vec{\theta}=\vec{\phi}^{*}$. Next, we will prove the three characteristics of $\vec{\phi}^{*}$.

Considering Eq. (6), Eq. (9) can be transformed into

$$
\omega_{i}=K^{*} r^{*} \sin \phi_{i}^{*}, \quad \forall i \in \mathcal{I}_{N},
$$

where $r^{*}$ is calculated by multiplying $e^{-i \psi^{\xi}}$ on both sides of Eq. (3), namely,

$$
r^{*}=\frac{1}{N} \sum_{j=1}^{N} \cos \left(\theta_{j}^{\xi}-\psi^{\xi}\right)=\frac{1}{N} \sum_{j=1}^{N} \cos \left(\phi_{j}^{*}\right) .
$$

We define $\Upsilon_{\vec{\phi}^{*}}=\left[\phi_{\min }^{*}, \phi_{\max }^{*}\right]$, where $\phi_{\min }^{*}\left(\phi_{\max }^{*}\right)$ is the minimum (maximum) $\phi^{*}$. We have two observations about $\Upsilon_{\vec{\phi}^{*}}$ : first, its length $\left|\Upsilon_{\vec{\phi}^{*}}\right|<\frac{\pi}{2}$ due to $\vec{\phi}^{*} \in \mathbb{S}_{K^{*}}^{\infty}$; secondly, $0 \in \Upsilon_{\vec{\phi}^{*}}$, because $\langle\omega\rangle=0$ implies $\omega_{\min } \leqslant 0$ and $\omega_{\max } \geqslant 0$, resulting in $\phi_{\min }^{*} \leqslant 0$ and $\phi_{\max }^{*} \geqslant 0$ from Eq. (14). These two 
characteristics of $\Upsilon_{\vec{\phi}^{*}}$ restrict $\Upsilon_{\vec{\phi}^{*}} \subset\left[-\frac{\pi}{2}, \frac{\pi}{2}\right]$. Thus, we get the analytical expression of the master solution $\left(\vec{\phi}^{*}\right)$ from Eq. (14),

$$
\phi_{i}^{*}=\arcsin \frac{\omega_{i}}{K^{*} r^{*}}, \quad \forall i \in \mathcal{I}_{N},
$$

which is independent of $\xi$, thus (i) and (ii) follows. From Eq. (14) we have $\sum_{i=1}^{N} \sin \phi_{i}^{*}=\frac{1}{K^{*} r^{*}} \sum_{i=1}^{N} \omega_{i}=0$. Substituting this into Eq. (5), we have $\psi^{*} \equiv 0$, which proves (iii).

Our goal is to find the master solution $\left(\vec{\phi}^{*}\right)$ as $K=K^{*} \geqslant K_{S}$ for the finite-size Kuramoto model. We know $\Upsilon_{\vec{\phi}^{*}} \subset\left[-\frac{\pi}{2}, \frac{\pi}{2}\right]$, implying $\cos \phi_{j} \geqslant 0, \forall j \in \mathcal{I}_{N}$. Thus, the order parameter in Eq. (15) is calculated as $r^{*}=$ $\frac{1}{N} \sum_{j=1}^{N} \sqrt{1-\sin \left(\phi_{j}^{*}\right)^{2}}$. Considering $\sin \phi_{j}^{*}=\omega_{j} /\left(K^{*} r^{*}\right)$ obtained from Eq. (14), $r^{*}$ can be expressed by a transcendental equation, namely,

$$
r^{*}=\frac{1}{N} \sum_{j=1}^{N} \sqrt{1-\left(\frac{\omega_{j}}{K^{*} r^{*}}\right)^{2}} .
$$

In order to obtain an approximate solution for $r^{*}$, we construct a new model for the original system. We relabel $\vec{\omega}$ such that $\omega_{1} \leqslant \omega_{2} \leqslant \cdots \leqslant \omega_{N}$, and split $\omega$ into two groups. One group is $\omega^{\prime}=\left[\omega_{1}, \omega_{2}, \ldots, \omega_{N^{\prime}}\right]^{T}$, where $N^{\prime}=\frac{N}{2}\left(N^{\prime}=\right.$ $\frac{N-1}{2}$ ) if $N$ is even (odd), and the other group is $\omega^{\prime \prime}=$ $\left[\omega_{N^{\prime}+1}, \omega_{N^{\prime}+2}, \ldots, \omega_{N}\right]^{T}$. We note that, since $\sum_{j=1}^{N^{\prime}} \omega_{j}+$ $\sum_{j=N^{\prime}+1}^{N} \omega_{j}=\sum_{j=1}^{N} \omega_{j}=0,\left\langle\omega^{\prime}\right\rangle=-\left\langle\omega^{\prime \prime}\right\rangle \leqslant 0$ when $N$ is even, and $\left\langle\omega^{\prime}\right\rangle \approx-\left\langle\omega^{\prime \prime}\right\rangle \leqslant 0$ when $N$ is odd. For simplicity, we indistinctly denote $\left\langle\omega^{\prime}\right\rangle \approx-\left\langle\omega^{\prime \prime}\right\rangle$ for both cases.

When all of the oscillators emerge into stable FS, our model treats the whole system as two oscillators in stable FS. The natural frequencies of the two oscillators are $\left\langle\omega^{\prime}\right\rangle$ and $\left\langle\omega^{\prime \prime}\right\rangle$. The two oscillators also follow the original FS Kuramoto model equations, namely,

$$
\begin{gathered}
\left\langle\omega^{\prime}\right\rangle=K^{*} r^{* \prime} \sin \phi^{\prime}, \\
\left\langle\omega^{\prime \prime}\right\rangle=K^{*} r^{* \prime} \sin \phi^{\prime \prime},
\end{gathered}
$$

where $r^{* \prime}$ is the order parameter for the two oscillators. The two oscillators are in stable FS, thus we have $\phi^{\prime}, \phi^{\prime \prime} \in\left[-\frac{\pi}{2}, \frac{\pi}{2}\right]$, which is obtained from the analysis of the Lyapunov function [Eq. (11)] for the two oscillators. Then the order parameter is $r^{* \prime}=1 / 2\left(\cos \phi^{\prime}+\cos \phi^{\prime \prime}\right)=$ $1 / 2 \sqrt{1-\left[\left\langle\omega^{\prime}\right\rangle /\left(K^{*} r^{*}\right)\right]^{2}}+1 / 2 \sqrt{1-\left[\left\langle\omega^{\prime \prime}\right\rangle /\left(K^{*} r^{*}\right)\right]^{2}}$. Further considering $\left|\left\langle\omega^{\prime}\right\rangle\right| \approx\left|\left\langle\omega^{\prime \prime}\right\rangle\right|$ we have

$$
r^{* \prime} \approx \sqrt{1-\left(\frac{\left\langle\omega^{\prime}\right\rangle}{K^{*} r^{*}}\right)^{2}}
$$

whose solution is

$$
\begin{aligned}
& r_{1}^{* \prime} \approx \lambda_{1}=\frac{\sqrt{2}}{2} \sqrt{1+\sqrt{1-\frac{4\left\langle\omega^{\prime}\right\rangle^{2}}{K^{* 2}}}}, \quad K^{*} \geqslant 2\left|\left\langle\omega^{\prime}\right\rangle\right|, \\
& r_{2}^{* \prime} \approx \lambda_{2}=\frac{\sqrt{2}}{2} \sqrt{1-\sqrt{1-\frac{4\left\langle\omega^{\prime}\right\rangle^{2}}{K^{* 2}}}}, \quad K^{*} \geqslant 2\left|\left\langle\omega^{\prime}\right\rangle\right|,
\end{aligned}
$$

where we have $\lambda_{1} \lambda_{2}=\frac{-\left\langle\omega^{\prime}\right\rangle}{K^{*}} \approx \frac{\left\langle\omega^{\prime \prime}\right\rangle}{K^{*}}$. Substituting this condition into Eqs. (18) and (19), we get $-\lambda_{1} \lambda_{2}=r^{* \prime} \sin \phi^{\prime}$ and $\lambda_{1} \lambda_{2}=r^{* \prime} \sin \phi^{\prime \prime}$. If $r^{* \prime} \approx \lambda_{2}$, we have $\phi^{\prime} \approx-\arcsin \left(\lambda_{1}\right)$ and $\phi^{\prime \prime} \approx \arcsin \left(\lambda_{1}\right)$. Because $\frac{\sqrt{2}}{2} \leqslant \lambda_{1} \leqslant 1$, we approximately have $-\frac{\pi}{2} \leqslant \phi^{\prime} \leqslant-\frac{\pi}{4}$ and $\frac{\pi}{4} \leqslant \phi^{\prime \prime} \leqslant \frac{\pi}{2}$. This means $\mid \phi^{\prime}-$ $\phi^{\prime \prime} \mid \geqslant \frac{\pi}{2}$, and $\lambda_{1}$ grows larger as $K$ increases from $K_{S}$ resulting in a growth of $\left|\phi^{\prime}-\phi^{\prime \prime}\right|$. However, $\left|\phi^{\prime}-\phi^{\prime \prime}\right| \geqslant \frac{\pi}{2}$ implies instability of the FS solution of the two oscillators, which can be understood by the Lyapunov function in Eq. (11) for the two oscillators. This means that $r^{* \prime} \approx \lambda_{2}$ describes an unstable FS solution. On the other hand, $r^{* \prime} \approx \lambda_{1}$ ensures the stability of the FS solution. Thus, we let $r^{*} \approx r^{* \prime} \approx \lambda_{1}$ be the approximation for the order parameter in Eq. (17), then the approximation $\left(\vec{\phi}^{* *}\right)$ for the master solution $\left(\vec{\phi}^{*}\right)$ in Eq. (16) is

$$
\phi_{i}^{* *}=\arcsin \frac{\omega_{i}}{K^{*} \lambda_{1}}, \quad \forall i \in \mathcal{I}_{N}
$$

Consequently, the approximate stable solution of the finite-size Kuramoto model in FS is

$$
\theta_{i} \approx \arcsin \frac{\omega_{i}}{K^{*} \lambda_{1}}+\xi, \quad \forall i \in \mathcal{I}_{N}, \quad \forall \xi \in \mathbb{R} .
$$

We use $\vec{\epsilon}$ with element $\epsilon_{i}=\left|\phi_{i}^{* *}-\phi_{i}^{*}\right|, \forall i \in \mathcal{I}_{N}$, to denote the absolute error between the approximate master solution $\left[\vec{\phi}^{* *}\right.$ in Eq. (23)] and the numerical one $\left[\vec{\phi}^{*}\right.$ in Eq. (16)], and $\sigma$ to denote the standard deviation of $\vec{\epsilon}$, defined as $\sigma=\sqrt{\frac{1}{N} \sum_{i=1}^{N}\left(\epsilon_{i}-\langle\epsilon\rangle\right)^{2}}$. To demonstrate the effectiveness of our method for a network with four oscillators, we show numerical results in Fig. 1. $\epsilon_{i}$ is large $\forall i \in \mathcal{I}_{N}$, when $K<K_{S}$ (a)
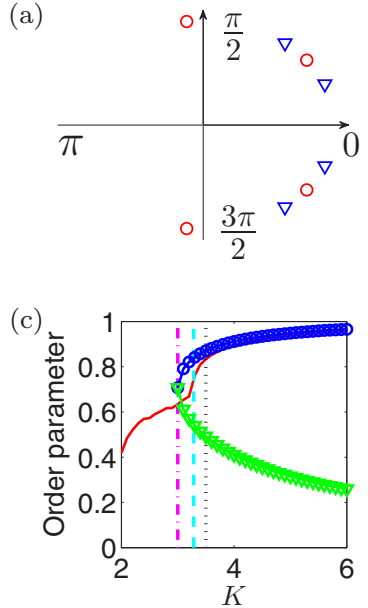

(b)

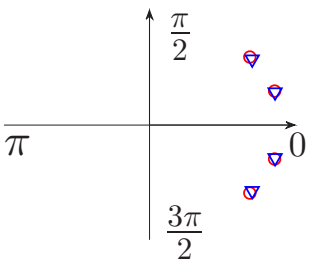

(d) 10

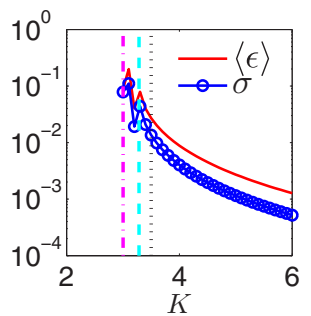

FIG. 1. (Color online) Results for a network of four oscillators with the vector of natural frequency given by $\vec{\Omega}=[-2,-1,1,2]^{T}$. (a) $K<K_{S}$; the blue triangles and red circles indicate the approximate master solution $\left(\vec{\phi}^{* *}\right)$ in Eq. (23) and the numerical one $\left(\vec{\phi}^{*}\right)$ in Eq. (16), respectively. (b) $K=K_{S} ; \vec{\phi}^{* *}$ indicated by blue triangles is close to $\vec{\phi}^{*}$ indicated by red circles. (c) The change of the order parameter and its approximation with respect to $K$. The red solid line indicates the numerical result of the order parameter in Eq. (17) (an average value of results from 2000 simulations with different initial phases). The blue solid line with circles indicates the change of $\lambda_{1}$ in Eq. (21), and the green solid line with triangles represents $\lambda_{2}$ in Eq. (22), as $K \geqslant 2\left|\left\langle\omega^{\prime}\right\rangle\right|$, where $2\left|\left\langle\omega^{\prime}\right\rangle\right|$ is the lowest bound of $K$ in Eqs. (21) and (22). The dash-dotted magenta line, dashed cyan line, and dotted black line represent $2\left|\left\langle\omega^{\prime}\right\rangle\right|, K_{C}$, and $K_{S}$, respectively. (d) The change of the average of the absolute error (red solid line) and standard deviation of the absolute error (blue solid line with circles) between $\vec{\phi}^{* *}$ and $\vec{\phi}^{*}$ as a function of $K$ when $K \geqslant 2\left|\left\langle\omega^{\prime}\right\rangle\right|$. 

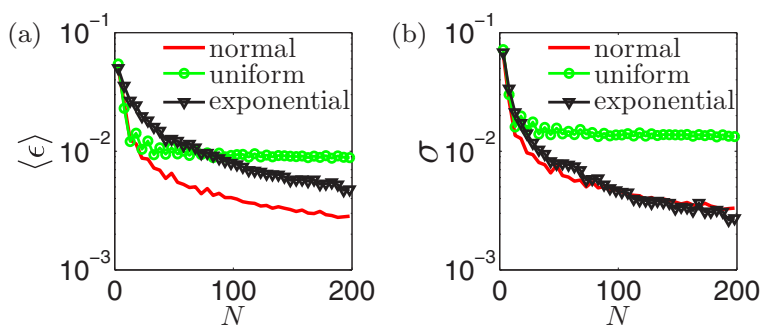

FIG. 2. (Color online) Study of the average absolute error $\langle\epsilon\rangle$ in (a), and the standard deviation $\sigma$ in (b) between the approximate master solution $\left(\vec{\phi}^{* *}\right)$ and the numerical one $\left(\vec{\phi}^{*}\right)$ at $K=K_{S} . N$ increases from 3 to 200 . The red line, green line with circles, and black line with triangles correspond to the normal distribution, uniform distribution, and exponential distribution of $\vec{\Omega}$, respectively. Results are based on the average value of results from 100 simulations for each distribution.

$\left[\vec{\phi}^{* *} \neq \vec{\phi}^{*}\right.$ in Fig. 1(a)], and small when $K=K_{S}\left[\vec{\phi}^{* *} \approx \vec{\phi}^{*}\right.$ in Fig. 1(b)]. The approximate order parameter, $\lambda_{1}$, is close to the numerical one as $K \geqslant K_{S}$ [Fig. 1(c)]. The average absolute error and standard deviation of the absolute error are small $\left(\langle\epsilon\rangle<10^{-1}, \sigma<10^{-1}\right.$ ) when $K \geqslant K_{S}$, and decreases rapidly as $K$ is increased [Fig. 1(d)]. This means that our method can calculate the master solution almost exactly in an analytical way for this network.

Figure 2 indicates that our method works well for networks with different $\vec{\Omega}$ distributions (uniform, normal, and exponential) and different number of oscillators ( $N$ increasing from 3 to 200). We obtain a uniform distribution $g(\Omega)$ by generating random numbers between -3 and 3 , a normal distribution by generating numbers following the probability density function $g(\Omega)=\frac{1}{\varrho \sqrt{2 \pi}} e^{-(\Omega-\mu)^{2} /\left(2 \varrho^{2}\right)}$ with the mean of the distribution $\mu=0$ and the standard deviation $\varrho=3$, and an exponential distribution by generating numbers following the probability density function $g(\Omega)=\frac{1}{\beta} e^{-\Omega / \beta}$, if $\Omega \geqslant 0$ and $g(\Omega)=0$, if $\Omega<0$, with the mean of the distribution $\beta=2$. We observe that $\langle\epsilon\rangle$ and $\sigma$ are larger when $N$ is smaller, but they decrease quickly as $N$ increases. Furthermore, $\langle\epsilon\rangle$ and $\sigma$ in Fig. 2 are obtained at $K=K_{S}$, meaning that they are the largest value obtained for each simulation. In other words, smaller $\langle\epsilon\rangle$ and $\sigma$ can be obtained if we increase $K$ for any given $N$ and any $\vec{\Omega}$ distribution, since larger $K$ implies smaller absolute error between the approximate order parameter and the numerical one as shown in Fig. 1(c), which further implies smaller absolute error between $\vec{\phi}^{* *}$ and $\vec{\phi}^{*}$.

\section{STABILITY OF THE SOLUTION}

A sufficient condition to ensure the stability of the FS solution was proposed by [9] as

$$
K>K_{P}, \quad \text { where } K_{P}=\frac{\sqrt{2}\left|\omega_{i}\right|}{r}, \quad \forall i \in \mathcal{I}_{N} .
$$

Taking $\lambda_{1}$ in Eq. (21) as the approximation of $r$, we get a sufficient condition for the stability of the FS solution, namely,

$$
K>K_{A}, \quad \text { where } K_{A}=\frac{\sqrt{2}\left|\omega_{i}\right|}{\lambda_{1}}, \quad \forall i \in \mathcal{I}_{N} .
$$
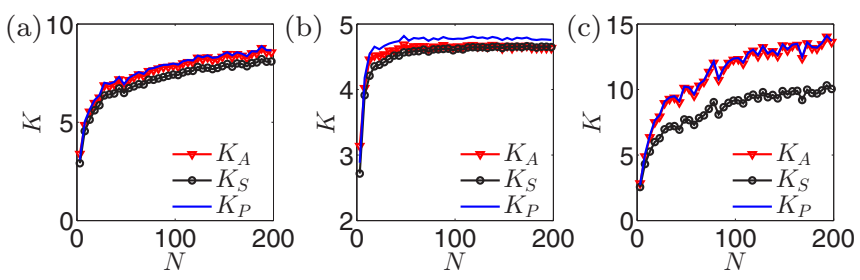

FIG. 3. (Color online) The stable critical coupling strength of the finite-size Kuramoto model. $N$ increases from 3 to 200. $K_{S}$ (black line with circles) is calculated by Eq. (12), $K_{P}$ (blue line) by Eq. (25), and $K_{A}$ (red line with triangles) by Eq. (26). (a) $\vec{\Omega}$ follows a normal distribution. (b) $\vec{\Omega}$ follows a uniform distribution. (c) $\vec{\Omega}$ follows an exponential distribution. The figures are drawn based on average results of 30 simulations for each distribution.

Equation (26) is useful to forecast whether the system is able to get into a stable FS state in an analytical way, without solving the differential equations. In other words, it reveals the relationship between the physical parameters (average natural frequency and coupling strength) and the stable behavior of oscillator networks described by the finite-size Kuramoto model explicitly.

Figure 3 shows the effectiveness of our condition to gauge the stability for the FS solution of the finite-size Kuramoto model, considering different $\vec{\Omega}$ distributions and different number of oscillators. If $\vec{\Omega}$ obeys a normal or exponential distribution [Figs. 3(a) and 3(c), respectively], $K_{A}$ in Eq. (26) coincides remarkably with $K_{P}$ in Eq. (25), and $K_{A}>K_{S}$. If $\vec{\Omega}$ obeys a uniform distribution [Fig. 3(b)], $K_{A}$ in Eq. (26) is close to $K_{S}$ in Eq. (12). This means Eq. (26) is effective to approximately determine the stability for the FS solution of the finite-size Kuramoto model.

\section{CONCLUSION}

In this paper, we studied the finite-size Kuramoto model [Eq. (2)], including its analytical solution for FS and its stable behavior. Our approximate frequency synchronization (FS) solution takes a simple form [Eq. (24)], which, surprisingly, is independent of the network size. These significant results are a consequence of a mathematical insight expressed in Eq. (16) and a physical insight in the model leading to Eqs. (18) and (19). Among an infinite number of FS solutions, we have understood that there is a particular one, the master solution in Eq. (16), which allows one to calculate all of the others. We have also understood that the FS in the finite-size Kuramoto model is approximately characterized by two clusters of oscillators that can be effectively described as two coupled oscillators in Eqs. (18) and (19). Furthermore, we developed a condition to approximately predict the stability for the FS solution of the finite-size Kuramoto model in an analytical way. This condition allows one to easily infer the relationship between the physical parameters and the stable behavior of networks.

\section{ACKNOWLEDGMENT}

C.W. acknowledges the studentship from the College of Physical Sciences, University of Aberdeen. N.R. acknowledges PEDECIBA, Uruguay. M.S.B. acknowledges EPSRC Grant No. EP/I032606/1. 
[1] M. Girvan and M. Newman, Proc. Natl. Acad. Sci. USA 99, 7821 (2002).

[2] G. Tononi, O. Sporns, and G. M. Edelman, Proc. Natl. Acad. Sci. USA 96, 3257 (1999).

[3] G. Robins, P. Pattison, Y. Kalish, and D. Lusher, Soc. Netw. 29 , 173 (2007).

[4] K. Langendoen and N. Reijers, Comput. Netw. 43, 499 (2003).

[5] A. R. Kiremire, M. R. Brust, and V. V. Phoha, Comput. Netw. 72, 14 (2014).

[6] P. H. Nardelli, N. Rubido, C. Wang, M. S. Baptista, C. PomalazaRaez, P. Cardieri, and M. Latva-aho, Eur. Phys. J. Spec. Top. 223, 2423 (2014).

[7] R. Carareto, M. S. Baptista, and C. Grebogi, Commun. Nonlin. Sci. Numer. Simul. 18, 1035 (2013).

[8] M. Verwoerd and O. Mason, SIAM J. Appl. Dyn. Syst. 7, 134 (2008).

[9] D. Aeyels and J. A. Rogge, Prog. Theor. Phys. 112, 921 (2004).

[10] F. Varela, J. Lachaux, E. Rodriguez, and J. Martinerie, Nat. Rev. Neurosci. 2, 229 (2001).

[11] M. G. Rosenblum, A. S. Pikovsky, and J. Kurths, Phys. Rev. Lett. 76, 1804 (1996).

[12] N. Wiener, Nonlinear Problems in Random Theory (Technology Press, New York, 1958).

[13] Y. Kuramoto, in International Symposium on Mathematical Problems in Theoretical Physics (Springer, New York, 1975), p. 420.

[14] Y. Kuramoto, Chemical Oscillations, Turbulence and Waves (Springer, Berlin, 1984).

[15] Y. Kuramoto and I. Nishikawa, J. Stat. Phys. 49, 569 (1987).

[16] H. Hong, M. Y. Choi, and B. J. Kim, Phys. Rev. E 65, 026139 (2002).
[17] Y. Moreno and A. F. Pacheco, Europhys. Lett. 68, 603 (2004).

[18] A. Politi and M. Rosenblum, Phys. Rev. E 91, 042916 (2015).

[19] J. A. Rogge and D. Aeyels, J. Phys. A 37, 11135 (2004).

[20] S. Luccioli and A. Politi, Phys. Rev. Lett. 105, 158104 (2010).

[21] A. Jadbabaie, N. Motee, and M. Barahona, in Proceedings of the American Control Conference 2004 (IEEE, Boston, 2004), Vol. 5, pp. 4296-4301.

[22] J. C. Bronski, L. DeVille, and M. J. Park, Chaos 22, 033133 (2012).

[23] G. Filatrella, A. H. Nielsen, and N. F. Pedersen, Eur. Phys. J. B 61, 485 (2008).

[24] P. Barbara, A. B. Cawthorne, S. V. Shitov, and C. J. Lobb, Phys. Rev. Lett. 82, 1963 (1999).

[25] V. Vlasov and A. Pikovsky, Phys. Rev. E 88, 022908 (2013).

[26] K. Wiesenfeld, P. Colet, and S. H. Strogatz, Phys. Rev. E 57, 1563 (1998).

[27] K. Wiesenfeld, P. Colet, and S. H. Strogatz, Phys. Rev. Lett. 76, 404 (1996).

[28] A. N. Grib, P. Seidel, and J. Scherbel, Phys. Rev. B 65, 094508 (2002).

[29] S.-Z. Lin, X. Hu, and L. Bulaevskii, Phys. Rev. B 84, 104501 (2011).

[30] G. Filatrella, N. F. Pedersen, and K. Wiesenfeld, Phys. Rev. E 61, 2513 (2000).

[31] J. A. Acebrón, L. L. Bonilla, C. J. P. Vicente, F. Ritort, and R. Spigler, Rev. Mod. Phys. 77, 137 (2005).

[32] N. Chopra and M. W. Spong, in Proceedings of the 44th IEEE Conference on Decision and Control, 2005 and 2005 European Control Conference (IEEE, Seville, 2005), pp. 3916-3922. 